\title{
Die Öffentlichkeit der Plattformen: Wechselseitige (Re-)Institutionalisierung von Öffentlichkeiten und Plattformen
}

\author{
Christian Katzenbach
}

Preprint

März 2020

Erscheint in: Eisenegger/Blum/Ettinger/Prinzing (Hrsg.) (2020): Digitaler Strukturwandel der Öffentlichkeit. Historische Verortung, Modelle und Konsequenzen. Wiesbaden: VS Verlag.

\section{Einführung: Der Aufstieg der Plattformen - und ihre Kontingenz}

Wir befinden uns inmitten eines tiefgreifenden Wandels sozialer Kommunikation. Soziale Medien und Plattformen sind selbstverständliche Mittel privater wie gesellschaftlicher Kommunikation und übernehmen in wachsendem Maße auch Vermittlungsleistungen von klassischen journalistischen Medien. ${ }^{1}$ Die Nutzung von Kommunikationsangeboten hat sich rapide zugunsten digitaler, meist mobiler Formen verschoben (vgl. überblickshaft Newman et al. 2019). Im Ergebnis haben sich die Dynamiken von Kommunikation und Öffentlichkeit verschoben und verstärkt.

\footnotetext{
${ }^{1}$ Die Begriffe „Soziale Medien“ und „Plattformen“ sind weder trennscharf noch unumstritten. Hier wird weitgehend dem Verständnis von Monika Taddicken und Jan-Hinrik Schmidt (2017: 8) gefolgt, wonach als Soziale Medien übergreifend alle „Angebote auf Grundlage digital vernetzter Technologien [verstanden werden], die es Menschen ermöglichen, Informationen aller Art zugänglich zu machen und davon ausgehend soziale Beziehungen zu knüpfen und/oder zu pflegen." Plattformen sind dann neben Wikis, Messaging- und Personal-Publishing-Diensten eine Untergruppe sozialer Medien, die eine Infrastruktur für Kommunikation bieten und die Inhalte von Nutzer'innen veröffentlichen und kuratieren (Taddicken/Schmidt 2018: 10; Gillespie 2018: 18). Gleichzeitig beschreibt der PlattformBegriff eine Entwicklung, die über den Sektor von Medien und Kommunikation hinausweist. Auch in Feldern wie Verkehr, Gesundheit und Immobilien etablieren sich neue Akteure, die vor allem Vermittlungsleistungen übernehmen, technische Infrastrukturen zur Verfügung stellen und Daten sammeln und analysieren (Poell/Nieborg/Van Dijck 2019). In diesem Artikel geht es um Plattformen sozialer Kommunikation als Untergruppe sozialer Medien.
} 
Dieser Wandel ist aufs Engste verbunden mit Irritationen und Kontroversen über die Regeln und Formen der gesellschaftlichen Kommunikation: Wie sollten Plattformen wie Facebook und YouTube mit Hate Speech und Misinformation umgehen? Wie lassen sich Privatsphäre und Datenschutz noch gewährleisten angesichts der massiven lukrativen Datenmengen, die wir alle durch die Nutzung von digitalen Endgeräten und Diensten andauernd erzeugen? Dass der Wandel mit Konflikten verbunden ist, verwundert nicht: In dem Maße, wie sich unsere Routinen der personalen und gesellschaftlichen Kommunikation ändern, können sich auch die Erwartungen an kommunikatives Handeln verschieben. Oft müssen sich neue Erwartungen und Regeln erst herausbilden, weil sich die neuen Angebote und Kommunikationsformen unseren etablierten Schemata entziehen: Ist das Posten von Tweets auf Twitter oder Hochladen eines Bildes in das Netzwerk meiner Freunde auf Facebook als private oder als öffentliche Kommunikation zu werten? Sind Plattformen wie YouTube und Facebook verantwortlich für die Inhalte ihrer Nutzer? Sind sie Medien oder neutrale Kanäle? Unterscheidungen wie die zwischen privat und öffentlich, zwischen Medienproduktion und Mediennutzung waren immer schon Vereinfachungen. Die Strukturen und Praktiken der gegenwärtigen digital vernetzten Kommunikation stellen sie aber in besonderem Maße in Frage. Damit verlieren auch die an solche Unterscheidungen gebundenen Regeln ihre Selbstverständlichkeit.

Zusammengenommen sind diese Umbrüche und Irritationen starke Indikatoren dafür, dass wir uns inmitten eines fundamentalen institutionellen Wandels der gesellschaftlichen Kommunikation befinden (Jarren 2019; vgl. Eisenegger und Jarren in diesem Band). In dem Maße, in dem Selbstverständlichkeiten aufbrechen, wächst der Bedarf an der Klärung von Rollen und an der Aushandlung von Regeln. Derzeit werden sowohl die Strukturen gesellschaftlicher Kommunikation re-institutionalisiert wie auch die Regeln neu verhandelt.

Im Kern dieses Wandels und der Debatte darüber stehen dabei die dominanten Internet-Anbieter Google, Facebook und Amazon, die über ihre Dienste und Plattformen maßgeblich gesellschaftliche Kommunikation vermitteln und strukturieren. Die Kommunikationswissenschaft hat diesen Umbruch in den vergangenen Jahren ernst genommen und intensiv beforscht. Es wird immer deutlicher, auf welche Weise Plattformen Kommunikation re-organisieren. Die Plattformen selbst werden dabei in der Regel als externe Kräfte verstanden, die sowohl von außen auf etablierte Medienstrukturen und bestehende Öffentlichkeiten einwirken als auch selbst neue Strukturen und Kommunikationsdynamiken begründen (Jarren 2019: 173). Dies ist richtig - allerdings gerät ein zentraler Aspekt dieses tiefgreifenden Wandels dabei aus dem Blick: Es wandeln sich in diesem Prozess nicht nur Medienstrukturen und Öffentlichkeiten, sondern auch die Plattformen. Die neuen Plattformen selbst sind kontingent - mit anderen Worten: Sie 
könnten auch anders sein. Ihr Entwicklung (und damit sowohl ihre gegenwärtige Gestalt wie auch ihre zukünftige!) hängt nicht einfach von einem irgendwie vorbestimmten Pfad technischen Fortschritts ab. Sie wird primär von den ökonomischen Interessen der Plattformen bestimmt, gleichzeitig aber auch maßgeblich von gesellschaftlichen und politischen Impulsen geprägt. Seit Anbeginn erfinden sich diese Plattformen beständig neu, ändern ihre Leitbilder wie ihre konkrete Ausgestaltung. Im Ergebnis sind sie weder als gesellschaftliche Akteure noch als Kommunikationsstrukturen Monolithen, die als unveränderliche Elemente von außen auf Gesellschaft und Medien einwirken. Vielmehr sind sie selbst Gegenstand eines andauernden und strittigen Institutionalisierungsprozesses. Welche Regeln gelten in diesen Kommunikationsumgebungen? Wer kann diese Regeln legitimerweise setzen? Und ganz grundsätzlich, was sind sie überhaupt: Medien oder neutrale Vermittler? In der Adressierung dieser Fragen agieren die Unternehmen natürlich selbst als machtvolle Akteure - gleichwohl ist dieser Wandlungsprozess auch gesellschaftlich und politisch gestaltbar. Welche Form die Plattformen als neue Institutionen der gesellschaftlichen Kommunikationen annehmen werden, wird letztlich auch durch Regulierung, öffentliche Kontroversen und Interventionen der Nutzer/innen entschieden.

Der Beitrag schlägt somit vor, den gegenwärtigen tiefgreifenden Wandel der gesellschaftlichen Kommunikation als einen Prozess zu verstehen, der maßgeblich durch die wechselseitige (Neu-)Institutionalisierung von Öffentlichkeiten und Plattformen geprägt ist. Dazu wird zunächst eine institutionentheoretische Perspektive auf Medienwandel und Plattformen eingeführt, die an die jüngere Wiederentdeckung der Institutionentheorie in der Kommunikationswissenschaft anknüpft. Mithilfe dieses Rahmens wird dann zuerst knapp die intensive und umfassende Forschung zum Öffentlichkeitswandel resümiert und die von Plattformen mitgestaltete Neu-Institutionalisierung von Öffentlichkeit rekonstruiert. Darauf folgt die andere Facette der wechselseitigen Institutionalisierung: Die Institutionalisierung von Plattformen wird nachgezeichnet als nicht-linearer Prozess, der nicht allein von technischen und ökonomischen Überlegungen der Plattformen abhängt, sondern ebenso von politischen und gesellschaftlichen Kontroversen.

\section{Eine institutionentheoretische Perspektive auf Öffentlichkeiten und Plattformen im Wandel}

Medien und Öffentlichkeiten sind gesellschaftliche Institutionen. Diese Perspektive ist in der Kommunikationswissenschaft zwar grundsätzlich weit verbreitet und auch im kanonischen Verständnis von Medien mit Saxer (1980; 1999: 6) als "komplexe institutionalisierte Systeme um organisierte Kommunikationskanäle 
von spezifischem Leistungsvermögen“ verankert. Waren Institutionentheorien aber bislang wenig sichtbar im empirischen und konzeptuellen Mainstream des Faches, lässt sich in der Auseinandersetzung mit dem gegenwärtigen tiefgreifenden Medienwandel eine jüngere Wiederentdeckung von Institutionentheorien in der Kommunikationswissenschaft ausmachen (Jarren 2019, Sandhu 2018, Katzenbach 2017, Künzler et al. 2013), die an bestehende Traditionslinien anknüpfen kann (Kiefer 2010, Donges 2006, Jarren/Steininger 2016).

Institutionen werden im Kontext verschiedener Strömungen institutioneller Theorien unterschiedlich betrachtet: sind Institutionen im Historischen Institutionalismus und im ökonomischen Neo-Institutionalismus zuvorderst Organisationen, Gesetze und andere formale Regelwerke, inkludieren soziologische Varianten des Neo-Institutionalismus auch Erwartungen und Diskurse in den Institutionenbegriff (vgl. Hall/Taylor 1996; DiMaggio 1998; als Überblick Katzenbach 2017: 88-96). Im Anschluss an die soziologischen Institutionentheorien werden im Beitrag Institutionen zunächst allgemein als Regelungs- und Erwartungsstrukturen verstanden, die legitimes Handeln und Entscheiden begründen (Hasse/Krücken 2005: 7). Mit Blick auf Medien hat diese institutionelle Perspektive eine doppelte Funktion: Sie bietet erstens einen Rahmen für die Erklärung von Medien und Öffentlichkeit als Institution, und zweitens einen Rahmen für die Erklärung der Rolle von Medien und Öffentlichkeit für verschiedene andere Institutionen. Die Diskussion um die zweite Perspektive erfährt derzeit unter Begriffen wie kommunikativer Institutionalismus (Sandhu 2018, Cornelissen et al. 2015), diskursiver Institutionalismus (Schmidt 2008), aber auch durch die Verbindung mit Konzepten wie Governance (Katzenbach 2017: 293ff.) vermehrte Aufmerksamkeit. Im vorliegenden Beitrag geht es dagegen stärker um die erste Perspektive, die Medien, Öffentlichkeiten und Plattformen selbst als Institutionen versteht - auch wenn deutlich werden wird, dass auch und gerade hier natürlich die kommunikative Dimension der Institutionalisierung ebenfalls von großer Bedeutung ist.

Blickt man mit der institutionentheoretischen Brille nun auf Medien, dann richtet sich der Blick auf der Mikro-Ebene etwa auf habituelle Formen der Mediennutzung, auf Erwartungen der Mediennutzer oder auf als legitim angesehene Handlungsweisen von Journalistinnen (z..B. Vertraulichkeit von Hintergrundgesprächen, Auswahl von Quellen); auf der Meso-Ebene auf geteilte professionelle Normen und Routinen, die sich etwa im Pressekodex und in der Etablierung verschiedener Ressorts und Textformate ausdrücken; auf der Makro-Ebene auf die Erwartungen, die wir an Medien etwa in einer Demokratie stellen (wie Vielfalt an Themen und Meinungen), oder auf die strukturelle Verfasstheit des Mediensystems (z.B. Wettbewerb, Öffentlich-Rechtliche-Medien) (vgl. Beck 2018: 26ff.). Damit wird deutlich, dass Institutionen auf mehreren Ebenen operieren - und nicht 
nur auf der Meso-Ebene der Organisation, auch wenn dieser Begriff mitunter mit dem der Institution vermischt wird. Gerade mit Blick auf die Medien sind Organisation und Institutionen aber sinnvollerweise zu unterscheiden: Die organisationale Perspektive bezieht sich auf das spezifische Organisieren von Kommunikation und das Schaffen von zweckrationalen Strukturen (Medienunternehmen). Institutionen manifestieren und zeigen sich zwar auch in diesen Organisationen, gehen darüber aber hinaus. „Sie geben dem individuellen Akteur Orientierung, stiften gesellschaftliche Ordnung und kollektiven Sinn.” (Beck 2018: 25).

Es hat sich als hilfreich erwiesen, diese durch Institutionen geleistete Orientierungs- und Ordnungsfunktion analytisch auf vier Ebenen zu betrachten (Scott 2007, Donges 2006, Puppis 2010 - vgl. zur Ergänzung der vierten Ebene Katzenbach 2017):

- $\quad$ auf der regulativen Ebene der Etablierung und Durchsetzung formaler Regeln;

- der normativen Ebene, in der Handlungsmöglichkeiten als legitim oder illegitim bewertet werden;

- der kulturell-kognitiven Ebene der oft unhinterfragten Konstruktion, Deutung und Wahrnehmung von sozialer Wirklichkeit und damit verbundenen Handlungsoptionen (taken-for-grantedness);

- $\quad$ sowie einer technologischen Ebene, auf der soziale Erwartungen und Regeln in technisch-materiellen Artefakte und Strukturen verkörpert, übersetzt und weiterentwickelt werden. ${ }^{2}$

Eine solche integrierte Perspektive macht deutlich, dass sich Ordnungs- und Wandlungsprozesse, etwa zu Regeln und Strukturen der medialen Kommunikation, über ganz unterschiedliche Mechanismen und an verschiedenen Orten realisieren. Neben Gesetzen und formalen Regeln stellen auch soziale Normen und geteilte Sichtweisen und Deutungsmuster kollektive Verbindlichkeit her, leisten die Koordination unterschiedlicher Interessen und bieten soziale Orientierung aber eben auch technisch-materielle Artefakte und Strukturen. Auf dieser institutionentheoretischen Basis lässt sich nun die Doppelbewegung wechselseitiger Institutionalisierung beschreiben: die einerseits stark von Plattformen getriebene ReInstitutionalisierung von Öffentlichkeit (Abschnitt 3) und die gleichzeitig von

\footnotetext{
${ }^{2}$ Mediale Kommunikation ist immer schon stark infrastrukturell verankert, die gegenwärtige tiefgreifenden Mediatisierung und Datafizierung der kommunikativen Verhältnisse lässt diese Betrachtung auch der technologischen Ebene umso wichtiger werden (Katzenbach 2017; Hepp 2016; vgl. Wimmer in diesem Band).
} 
Öffentlichkeit, Politik und mitunter auch Nutzer*innen mitgestaltete Institutionalisierung von Plattformen (Abschnitt 4).

\section{Die Öffentlichkeiten der Plattformen}

Plattformen tragen zum Wandel von Öffentlichkeit bei. Dieser Aspekt der wechselseitigen (Re-)Institutionalisierung von Öffentlichkeiten und Plattformen ist in der Kommunikationswissenschaft sehr präsent und wird intensiv beforscht. Unbestritten ist, dass sich im Zuge der Etablierung von Plattformen und Social-MediaDiensten die Vermittlungsformen gesellschaftlicher Kommunikation deutlich verändert haben: Journalistische Verfahren und Kriterien der Zuweisung von Relevanz werden zunehmend durch Algorithmen komplementiert, teilweise ersetzt im Übrigen nicht nur auf Plattformen, sondern auch in journalistischen Angeboten (Just/Latzer 2017, Loosen/Scholl 2017). Christoph Neuberger (2004, 2009) hat schon in den 2000er Jahren darauf hingewiesen, dass neben die professionell-journalistische Vermittlung partizipative und technische Formen hinzutreten. Googles Suchmaschine organisiert Treffer nach mehr oder weniger personalisierten Relevanzkriterien; Facebooks Newsfeed zeigt uns Nutzer*innen Inhalte und Meldungen an, die möglichst viel „Engagement“, also Interaktionen auf der Plattform, erzeugen sollen (Bucher 2012, 2017); Twitter und Instagram kuratieren ebenfalls den Strom von Meldungen nach eigenen Kriterien. Dabei sind Plattformen nicht nur zu machtvollen Relevanzmaschinen, sondern auch zu entscheidenden Regulierungsmaschinen geworden (Katzenbach 2018): Sie treffen regelmäßig nicht nur Entscheidungen über die Relevanz von Inhalten, sondern auch darüber, ob Inhalte überhaupt auf der Plattform gezeigt werden sollen und dürfen.

Fasst man diesen Wandlungsprozess als eine Re-Institutionalisierung von Öffentlichkeiten, die maßgeblich durch Plattformen getrieben wird, lassen sich Veränderungen auf allen vier Ebenen identifizieren:

Auf der regulativen Ebene der Etablierung und Durchsetzung formaler Regeln mit Bezug zu Öffentlichkeiten treten neben traditionelle medienpolitische Regelungen wie das Presserecht, Staatsverträge zur Regelung des öffentlich-rechtlichen und privaten Rundfunks neue Formen der Regelung von Kommunikation, die zuvorderst von den Plattformen selbst stammen: In Community Guidelines, Datenschutz-Bestimmungen und Allgemeinen Geschäftsbedingungen (AGBs) setzen die Anbieter die Regeln für Kommunikation und Interaktionen unter den Nutzer/innen - und bestimmen damit maßgeblich Konturen öffentlicher Kommunikation: 
„,[A]s with broadcasting and publishing, their choices about what can appear, how it is organized, [...] what can be removed and why, and what the technical architecture allows and prohibits, are all real and substantive interventions into the contours of public discourse. They raise both traditional dilemmas about free speech and public expression, and some substantially new ones. " (Gillespie 2010: 359)

In Folge der jüngeren Debatten um Misinformation und Hate Speech ist diese zentrale Rolle der Plattformen offensichtlich geworden: Facebook und YouTube bestimmen, wo und entlang welcher Kriterien die Grenze zwischen legitimer Meinungsäußerung und unakzeptablen Inhalten verläuft - ob sie wollen oder nicht.

Damit verbunden ist ein Wandel auf der normativen Ebene von Öffentlichkeit als gesellschaftlicher Institution, in der Handlungsmöglichkeiten als legitim oder illegitim bewertet werden, sowohl mit Blick auf individuelle Kommunikationen von Einzelnen als auch auf die Rolle korporativer Akteure: Durch die Verschiebung der einfachen Öffentlichkeiten von Alltagsgesprächen und persönlichen Kommentaren in den medialen Raum (Katzenbach 2008, Neuberger 2009, Schmidt 2009) hat sich die Pluralität von veröffentlichten Meinungen und Äußerungsformen enorm erhöht. Veröffentlichte Kommunikation durchläuft nicht länger zwangsläufig die Routinen des professionellen Journalismus. So nimmt es nicht Wunder, dass sich die Grenzen der öffentlichen Meinungsäußerung nicht nur verschoben haben, sondern auch beständig umstritten sind. Die Debatten um Hate Speech und Misinformation zeigen, wie schwer diese Grenzziehung zwischen als legitim oder illegitim wahrgenommener Kommunikation prozessual wie substantiell fällt.

Auch auf der korporativen Ebene hat der Aufstieg der Plattformen zu zentralen Vermittlern sozialer Kommunikation eine Verschiebung normativer Erwartungen begünstigt. Die Plattformen haben sich zunächst stark außerhalb bestehender Medienstrukturen institutionalisiert und so auch neue Leitideen und Erwartungen sozialer Kommunikation etablieren können (Jarren 2019:172ff): u.a. Alltagsorientierung, Interaktion, Quantifizierung - denen sich inzwischen auch die etablierten Medieninstitutionen nicht verschließen können, wollen sie nicht an Legitimation verlieren (Jarren 2019; Schmidt/Loosen 2012).

Auf der kulturell-kognitiven Ebene der Organisation von Öffentlichkeit verschieben sich mit dem Aufstieg der Plattformen etwa die Mechanismen der Zuweisung von Relevanz. Mit der breiten Durchsetzung von Digitalisierung und Vernetzung haben der Journalismus und andere professionelle Medienakteure nicht mehr den exklusiven Zugriff auf die mediale Wirklichkeitskonstruktion (Neuberger 2009; Loosen/Scholl 2017). Die von Plattformen verfolgte grundsätzliche 
Operationalisierung von Relevanz als Interaktion ist, bei aller Komplexität und Intransparenz, so zu einem wesentlichen Strukturierungsmechanismus digitaler Kommunikation geworden. Diese "Social Media Logics" (Van Dijck/Poell 2013; Klinger/Svensson 2014) begünstigen kurze und kontroverse Inhalte und die Dynamiken rund um Hate Speech und Misinformation. Besonders YouTube wird intensiv für seinen Auto-Play-Algorithmus kritisiert, der regelmäßig zunehmend radikalere Inhalte empfiehlt (Kaiser/Rauchfleisch 2019). In diesen Kontroversen zeigt sich aber auch die Anpassungsfähigkeit der Plattformen (vgl. folgendes Kapitel): So werden Nachrichtenbeiträge und -videos von Facebook im andauernden Umbau ihres Systems mal als weniger, mal als stärker relevant klassifiziert. Während Facebook vor wenigen Jahren erst eingeführt hatte, dass Nachrichtenvideos eine besonders hohe Sichtbarkeit im Newsfeed bekommen, hat das Unternehmen in der Aufarbeitung der Misinformation-Diskussion beschlossen, Nachrichten insgesamt eine deutlich weniger prominente Rolle zuzuschreiben.

All diese Verschiebungen in der Organisation und Gestalt von Öffentlichkeiten hängen fraglos maßgeblich mit der technisch-infrastrukturellen Ebene von Öffentlichkeiten zusammen, teils lagern sich diese Verschiebungen in Technologien $\mathrm{ab}$, teils sind die Veränderungen getrieben von Technologien. Die Verbindung von Digitalisierung und Vernetzung, die breite Etablierung von Smartphones und der Aufbau von massiven Infrastrukturen (Daten- und Rechenzentren) haben diese Formen der algorithmischen Ordnung von Kommunikation (Loosen/Scholl 2017; Gorwa/Binns/Katzenbach 2020) erst möglich gemacht.

Zusammengefasst begünstigen Plattformen deutliche Verschiebungen auf der regulativen, normativen, kognitiv-kulturellen und technisch-infrastruktureller Ebene von Öffentlichkeiten als gesellschaftlicher Institution. Diese institutionellen Verschiebungen begründen Veränderungen in den kommunikativen Dynamiken, die sich derzeit in Diagnosen eines Wandel von Öffentlichkeit zeigen - wie etwa eine zunehmende Dynamisierung, Beschleunigung, Fragmentierung, und Legitimitätsbedürftigkeit von Öffentlichkeit. Auch wenn nicht all diese Diagnosen unumstritten sind, so ist doch offenkundig geworden, dass der Aufstieg der Plattformen maßgeblich zu einer Neu-Institutionalisierung von Öffentlichkeiten beigetragen hat.

\section{Die Plattformen der Öffentlichkeit}

Diese Impulse von Plattformen in der Neu-Institutionalisierung von Öffentlichkeiten konstituieren aber nur den einen Teil des Wechselverhältnisses von Plattformen und Öffentlichkeiten - zumal den deutlich häufiger beachteten. Bei dieser Betrachtung werden Plattformen als unveränderliche, stabile Elemente betrachtet - dabei sind sie das keineswegs. Sie befinden sich selbst in einem andauernden 
Aushandlungs- und Anpassungsprozess hinsichtlich sowohl ihrer Leitbilder wie ihrer konkreten Ausgestaltung. Der gegenwärtige tiefgreifende Wandel der gesellschaftlichen Kommunikation lässt sich nicht umfassend verstehen, ohne diese Veränderlichkeit und Kontingenz von Plattformen in den Blick zu nehmen. Mit anderen Worten: Wir befinden uns inmitten eines Prozesses der Institutionalisierung der Plattformen als zentralen Vermittlern gesellschaftlicher Kommunikation (Jarren 2019) - dieser Prozess ist aber weder bereits abgeschlossen, noch verläuft er linear oder selbstverständlich in eine bestimmte Richtung. Plattformen sind grundsätzlich kontingent und damit auch gestaltbar, durch die betreibenden Organisationen selbst, aber auch politisch und gesellschaftlich. Im Folgenden wird dieser wechselhafte Institutionalisierungsprozess von Plattformen exemplarisch entlang der vier Ebenen beschrieben.

Auf regulatorischer Ebene gestaltet sich der Institutionalisierungsprozess zwar im Detail durchaus wechselhaft, es zeichnet sich aber ein deutlicher übergreifender Trend ab: Plattformen werden zunehmend stärker für die von ihnen bereitgestellten Inhalte in Haftung genommen. Diese Entwicklung reflektiert auf regulatorischer Ebene den politischen und gesellschaftlichen Diskurs, der die Plattformen spätestens seit der US-Wahl 2016 und der Migrationsdebatte in Europa stärker in der Verantwortung für kommunikative und gesellschaftliche Entwicklungen sieht. Regulatorisch zeigt sich das in einer sukzessiven Abkehr vom sowohl in den USA als auch in Europa rund um die Jahrtausendwende etablierten "Noticeand-Take-Down"-Verfahren bzw. dem sogenannten Haftungsprivileg. Dieses Paradigma spricht Plattform-Anbieter grundsätzlich von der Haftung für Inhalte frei; erst nach Hinweis auf rechtswidrige Inhalte müssen diese entfernt werden. In einer Reihe von Gerichtsurteilen höchster Instanz sowie Gesetzgebungsinitiativen haben sich insbesondere europäische Institutionen von diesem Paradigma abgewendet und höhere Anforderungen und stärkere Haftungsregeln eingeführt. Kulminiert sind diese Entwicklungen bislang im deutschen Netzwerkdurchsetzungsgesetz (NetzDG) sowie in der neuen Europäischen Urheberrechtsrichtlinie, die beide Plattformen unter bestimmten Umständen direkt haftbar für Inhalte machen (Schulz 2018; Gorwa 2019; Katzenbach 2020).

Damit werden aber nicht nur einfach neue Regeln für bestimmte, existierende Akteurstypen gesetzt. Es sind gleichzeitig die Regulierung als auch die Anbieter selbst auf der Suche danach und in der Aushandlung darüber, was Plattformen eigentlich sind und welchem Akteurstypus sie entsprechen oder entsprechen sollten - also etwa Medienunternehmen, IT-Anbieter, Kabelnetzbetreiber. Das zeigt sich auch im Wandel der internen Regelsetzung der Anbieter selbst. Die AGBs und Community Guidelines der Plattformen sind keinesfalls so stabil wie es im vorangegangenen Kapitel erschienen haben mag, die Plattformen reagieren in diesen Dokumenten deutlich auf politische und öffentliche Kontroversen. Dabei 
lassen sich vor allem zwei Trends erkennen (Gollatz 2019): Die Anbieter haben (1.) die anfängliche rechtliche Aneignung der Nutzerinhalte schon früh umgestaltet in die verpflichtete Übereignung von Nutzungsrechten an den Inhalten - und damit versucht, die Verantwortung und Haftung bei den Nutzer/innen zu verorten. Zudem lässt sich (2.) im Zusammenhang mit dem wachsenden Druck auf Plattformen ein deutlicher langfristiger Trend aufzeigen, Inhalte auf den Plattformen stärker zu regulieren und die internen Regeln auszudifferenzieren (Katzenbach/Gollatz 2017). So hat etwa Facebook in seinen Community Guidelines die 2007 noch sehr breite Kategorie "inappropriate content" inzwischen aufgeteilt in zahlreiche, nun viel detaillierter beschriebene und begründete Themenfelder wie Hassrede, Gewalt und Nacktdarstellung. Facebook hat dabei zum Beispiel auf Proteste von stillenden Müttern und Familien reagiert, indem Fotos mit stillenden Frauen nun explizit als Ausnahme zum allgemeinen Verbot der Darstellung nackter weiblicher Brüste aufgeführt werden. Zudem hat die intensive Debatte um die Löschung des ikonischen Vietnamkrieg-Fotos "Terror of War" in einem Post Facebook zur Einführung von Abwägungsregeln veranlasst, die auch Kontextfaktoren von Bildern miteinbeziehen wie politische Relevanz und Satire. ${ }^{3}$ Im Gesamtbild zeigen sich damit deutliche Änderungen auf der regulatorischen Ebene von Plattformen, die auf den Charakter der Dienste selbst durchschlagen. Indem Plattformen anders reguliert werden und selbst Inhalte heute auch anders regulieren, sind sie zu etwas anderem geworden. Die Institutionalisierung von Plattformen wird also maßgeblich von Regulierung mitgestaltet. Mit anderen Worten: Regulierung erfüllt hier eine performative Wirkung. Die kuratorische Rolle von Plattformen, ihre Strukturierungswirkungen treten heute deutlich häufiger zu tage Tarleton Gillespie (2018) sieht in der Moderation von Inhalten gar das zentrale Angebot und Qualitätsversprechen von Plattformen.

Gleichzeitig haben sich auch die normativen Erwartungen an Plattformen massiv verschoben. Insgesamt wird ihnen heute nicht nur eine quantitativ und qualitativ höhere Relevanz für die Organisation gesellschaftlicher Kommunikation und damit mittelbar von Demokratie zugeschrieben - sondern auch eine deutlich höhere Verantwortung. Daher werden auch regelmäßig (und mit guten Gründen!) Maßnahmen eingefordert, um den für Demokratie und Öffentlichkeit dysfunktionalen Dynamiken wie Misinformations-Kampagnen in Wahlkämpfen und systematische Hassrede gegen Gruppen und Einzelne entgegen zu wirken. So zeigen sich Prozesse der Aushandlung darüber, was legitime Verfahrensweisen sind, auch in der Gestaltung des Newsfeeds, dem Kern von Facebooks Angebot. Der Dienst hat nicht nur regelmäßig Kriterien der Relevanzzuweisung verändert, sondern etwa in der Auswahl der "Trending Topics" bereits mehrmals zwischen rein

\footnotetext{
${ }^{3} \mathrm{https}$ ://de-de.facebook.com/communitystandards/objectionable_content.
} 
algorithmischen Auswahlprozessen einerseits und journalistischer Kuration gewechselt ${ }^{4}$ - jeweils in engem Zusammenhang mit öffentlichen Debatten über die Legitimität des jeweiligen Vorgehens.

Auf der kulturell-kognitiven Ebene der Deutung und Wahrnehmung zeigt sich der Wandel u.a. in der grundsätzlichen sozialen Konstruktion und Wahrnehmung von Plattformen. So betreibt etwa Facebook selbst eine andauernde kommunikative Neuerfindung, die mal von orientierenden Zukunftsbildern, mal von technischer und ökonomischer Entwicklung, mal von der öffentlichen Debatte getrieben wird (vgl. im Folgenden Haupt 2020): Die initiale Vision eines "online directory" (2004) und einer "social utility" (2005) wich mit dem rasanten Wachstum hin zu einer "social infrastructure" to "help people connect and communicate more efficiently" (2009). Mit dem Börsengang 2012 wird "global connectivity" zum zentralen Leitbild, zunächst quantitativ als Wachstum, ab 2015 wird es dann stärker qualitativ umgedeutet als starke, sichere Gemeinschaft - auch getrieben durch die öffentlichen und politischen Debatten um Hassrede und Misinformation. Damit einher geht auch die sich abzeichnende Abkehr von der lange recht erfolgreichen strategischen Positionierung als "neutraler Plattform" (Gillespie 2010, 2017) und die geringer werdende Abwehr, als Medienunternehmen bezeichnet zu werden (Napoli/Caplan 2017). In jüngeren Äußerungen sprechen Facebook und CEO etwa regelmäßig von der Verantwortung, die sie für Inhalte und Nutzer*innen haben. ${ }^{5}$

Auf technischer Ebene zeichnen sich Plattformen im Detail ohnehin durch ein andauerndes Experimentieren und Optimieren aus. Sie sind hoch komplexe und integrierte Infrastrukturen, deren spezifische Gestaltung und Optimierung zu großen Teilen durch ökonomische Interessen und mit Abstrichen durch regulatorische Rahmenbedingungen erklärt werden können (Helmond/Nieborg/Van der Vlist 2019). Dadurch wird die ökonomische und normative Orientierung dieser Optimierung regelmäßig neu ausjustiert etwa hinsichtlich der Relevanzkriterien im Newsfeed (vgl. Beispiel oben zur Rolle von Nachrichteninhalten).

Zusammengenommen zeigen diese Beispiele, wie wenig linear und eindeutig der Institutionalisierungsprozess der Plattformen auf allen vier Ebenen verläuft. Natürlich agieren sie selbst als äußerst machtvolle, vor allem von ökonomischen Interessen getriebenen Akteure in diesem Prozess. Sie sind aber keinesfalls Monolithe, die stabil und unveränderlich sind. Vielmehr sind sie in hohem Maße abhängig von gesellschaftlicher und politischer Akzeptanz und passen ihre Positionierung und ihre Aktivitäten daraufhin an.

\footnotetext{
${ }^{4}$ https://www.nytimes.com/2019/08/20/technology/facebook-news-humans.html

${ }^{5}$ Vgl. etwa Lischka (2019) mit einer Analyse der Anhörung Zuckerbergs vor dem Europäischen Parlament, sowie jüngere Blogposts des Unternehmens (Zuckerberg 2018; Harris 2020).
} 


\section{$5 \quad$ Fazit}

Der Beitrag hat vorgeschlagen, den gegenwärtigen tiefgreifenden Wandel der gesellschaftlichen Kommunikation als einen Prozess zu verstehen, der maßgeblich durch die wechselseitige (Neu-)Institutionalisierung von Öffentlichkeiten und Plattformen geprägt ist. Es ist offenkundig, dass die dominanten Internet-Anbieter wie Google, Facebook und Amazon zentrale Akteure dieses Wandels sind, indem sie über ihre Dienste und Plattformen maßgeblich gesellschaftliche Kommunikation vermitteln und strukturieren. In der bisherigen Forschung werden sie noch zu häufig als externe Kräfte verstanden, die Öffentlichkeiten und Kommunikationsdynamiken verändern. Dabei befinden sich die Plattformen selbst noch in einem Institutionalisierungsprozess, indem nicht nur ihre Integration in Gesellschaft und Politik unabgeschlossen ist, sondern auch ihre eigene Formation: Was sind Plattformen eigentlich und wie können wir sie gesellschaftlich mitgestalten und politisch regulieren? Vor diesem Hintergrund hat der Beitrag dafür plädiert, Plattformen nicht nur als externe stabile Faktoren zu betrachten. Sie sind selbst in höchstem Maße erklärungsbedürftig und hoch relevante Gegenstände kommunikationswissenschaftlicher Forschung. Der vorliegende Beitrag hat einen ersten Beitrag dazu geleistet, indem er ausschnittsweise den Wandel von Öffentlichkeiten und den Aufstieg der Plattformen als wechselseitigen Institutionalisierungsprozess rekonstruiert hat. In dieser Knappheit kann das nur ein kleiner erster Schritt im Abschreiten dieser Forschungsagenda zur Formation der Plattformen sein. Detaillierte Studien in diese Richtung werden wichtige Beiträge zur Erklärung des gegenwärtigen Wandels von Öffentlichkeit liefern. Wenn dieser Artikel zum Loslaufen auf diesem Weg angeregt hat, dann hat er seine Funktion erfüllt.

\section{Literatur}

Beck, Klaus (2018): Das Mediensystem Deutschlands: Strukturen, Märkte, Regulierung (2., überarbeitete und aktualisierte Auflage). Wiesbaden: Springer VS.

Bucher, Taina (2012): Want to Be on the Top? Algorithmic Power and the Threat of Invisibility on Facebook. In: New Media \& Society 14(7), S. 1164-80. https://doi.org/10.1177/1461444812440159.

Bucher, Taina (2017): The Algorithmic Imaginary: Exploring the Ordinary Affects of Facebook Algorithms. In: Information, Communication \& Society 20(1), S. 30-44. https://doi.org/10.1080/1369118X.2016.1154086.

Cornelissen, Joep P./Durand, Rodolphe/Fiss, Peer C./Lammers, John C./Vaara, Eero (2015): Putting Communication Front and Center in Institutional Theory and 
Analysis. In: Academy of Management Review 40 (1), S. 10-27. https://doi.org/10.5465/amr.2014.0381.

DiMaggio, Paul (1998): The new institutionalisms: avenues of collaboration. Journal of Institutional and Theoretical Economics 154 (4), S. 696-705.

Donges, Patrick (2006): Medien als Institutionen und ihre Auswirkungen auf Organisationen. Perspektiven des soziologischen Neo-Institutionalismus für die Kommunikationswissenschaft. Medien \& Kommunikationwissenschaft, 54, 563-578.

Gillespie, Tarleton (2010): The Politics of 'Platforms. In: New Media \& Society 12(3), S. 347-64. https://doi.org/10.1177/1461444809342738.

Gillespie, Tarleton (2017): The Platform Metaphor, Revisited. HIIG Digital Society Blog. URL: https://www.hiig.de/en/the-platform-metaphor-revisited/.

Gorwa, R. (2019). The platform governance triangle: Conceptualising the informal regulation of online content. Internet Policy Review, 8 (2). https://dx.doi.org/ 10.14763/2019.2.1407.

Gorwa, Robert/Binns, Reuben/Katzenbach, Christian (2020): Algorithmic content moderation: Technical and political challenges in the automation of platform governance. Big Data \& Society. https://dx.doi.org/10.1177/2053951719897945

Hall, Peter A./Taylor, Rosemary C. R. (1996): Political Science and the Three New Institutionalisms. Political Studies 44 (5), S. 936-957. doi: 10.1111/j.14679248.1996.tb00343.X.

Harris, Brent (2020): Preparing the Way Forward for Facebook's Oversight Board. Facebook Newsroom, 28.01.2020. URL: https://perma.cc/C7MM-V9WN.

Hasse, Raimund/Krücken, Georg (2005): Neo-Institutionalismus. Bielefeld: Transcript.

Haupt, Joachim (2020, im Erscheinen): Facebook Futures: Mark Zuckerberg's Discursive Construction of a Better World. New Media \& Society.

Helmond, Anne/Nieborg, David/van der Vlist, Fernando (2019): Facebook's evolution: Development of a platform-as-infrastructure. Internet Histories, 3(2), 123-146. doi: 10.1080/24701475.2019.1593667.

Hepp, Andreas (2016): Kommunikations- und Medienwissenschaft in datengetriebenen Zeiten. Publizistik, 61, 225-246.

Jarren, Otfried (2019): Fundamentale Institutionalisierung: Social Media als neue globale Kommunikationsinfrastruktur. In: Publizistik 64(2), S. 163-79. https://doi.org/10.1007/s11616-019-00503-4.

Jarren, Otfried/Steininger, Christian (2016): Journalismus jenseits von Markt und Staat: Institutionentheoretische Ansätze und Konzepte in der Publizistik- und Kommunikationswissenschaft. Baden-Baden: Nomos.

Just, Natascha/Latzer, Michael (2017): Governance by Algorithms: Reality Construction by Algorithmic Selection on the Internet. In: Media, Culture \& Society 39(2), S. 238 58. https://doi.org/10.1177/0163443716643157.

Kaiser, Jonas/Rauchfleisch, Adrian (2019). The implications of venturing down the rabbit hole. Internet Policy Review, 8(2).

Katzenbach, Christian (2017): Die Regeln digitaler Kommunikation. Governance zwischen Norm, Diskurs und Technik. Wiesbaden: Springer VS.

Katzenbach, Christian (2018): Die Ordnung der Algorithmen - Zur Automatisierung von Relevanz und Regulierung gesellschaftlicher Kommunikation. In R. M. Kar, B. Thapa 
und P. Parycek (Hrsg.), (Un)berechenbar? Algorithmen und Automatisierung in Staat und Gesellschaft, S. 315-338. Berlin: Kompetenzzentrum Öffentliche IT.

Katzenbach, Christian (2020): Die Governance sozialer Medien. In: Jan-Hinrik Schmidt/Monika Taddicken (Hrsg.), Handbuch Soziale Medien. Wiesbaden: Springer Fachmedien.

Katzenbach, Christian/Gollatz, Kirsten (2017): Platform Governance as Reflexive Coordination - Mediating Nudity, Hate Speech And Fake News On Facebook. Association of Internet Researchers (AoIR) 2017. Konferenzbeitrag. Tartu, Oktober 2017. https://doi.org/10.31235/osf.io/aj34w.

Kiefer, Marie-Luise (2010): Journalismus und Medien als Institutionen. Konstanz: UVK Verlagsgesellschaft.

Klinger, Ulrike/Svensson, Jakob (2015): The Emergence of Network Media Logic in Political Communication: A Theoretical Approach. In: New Media \& Society 17(8), S. 1241-57. https://doi.org/10.1177/1461444814522952.

Künzler, Matthias/Oehmer, Franziska/Puppis, Manuel/Wassmer, Christian (Hrsg.) (2013): Medien als Institutionen und Organisationen: Institutionalistische Ansätze in der Publizistik- und Kommunikationswissenschaft. Baden-Baden: Nomos.

Lischka, Juliane A. 2019. Strategic Communication as Discursive Institutional Work: A Critical Discourse Analysis of Mark Zuckerberg's Legitimacy Talk at the European Parliament. International Journal of Strategic Communication 13 (3), S. 197-213. doi: 10.1080/1553118X.2019.1613661.

Loosen, Wiebke/Schmidt, Jan-Hinrik (2012): (RE-)DISCOVERING THE AUDIENCE: The Relationship between Journalism and Audience in Networked Digital Media. In: Information, Communication \& Society 15(6), S. 867-87. https://doi.org/10.1080/1369118X.2012.665467.

Loosen, Wiebke/Scholl, Armin (2017): Journalismus Und (Algorithmische) Wirklichkeitskonstruktion. Epistemologische Beobachtungen. In: Medien \& Kommunikationswissenschaft 65(2), S. 348-66. https://doi.org/10.5771/1615-634X-2017-2-348.

Napoli, Philip/Caplan, Robyn (2017): Why Media Companies Insist They're Not Media Companies, Why They're Wrong, and Why It Matters. In: First Monday 22 (5). https://doi.org/10.5210/fm.v22i5.7051.

Neuberger, Christoph (2004): Wandel der aktuellen Öffentlichkeit im Netz. Gutachten für den Deutschen Bundestag. Berlin, 2004.

Neuberger, Christoph (2009): Internet, Journalismus und Öffentlichkeit. In Christoph Neuberger, Christian Nuernbergk und Melanie Rischke (Hrsg): Journalismus im Internet, S. 19-105. Wiesbaden: VS Verlag für Sozialwissenschaften. https://doi.org/10.1007/978-3-531-91562-3_2.

Newman, Nic/Fletcher, Richard/ Kalogeropoulos, Antonis/Nielsen, Rasmus Kleis (2019): Reuters Institute Digital News Report 2019. Reuters Institute for the Study of Journalism, Oxford.

Poell, Thomas/Nieborg, David/van Dijck, José (2019) : Platformisation. Internet Policy Review 8 (4). https://doi.org/10.14763/2019.4.1425.

Puppis, Manuel (2010): Media Governance: A New Concept for the Analysis of Media Policy and Regulation. In: Communication, Culture \& Critique 3 (2), S. 134-49. https://doi.org/10.1111/j.1753-9137.2010.01063.x. 
Sandhu, Swaran (2018): Kommunikativer Institutionalismus und Accounts. In Annika Schach und Cathrin Christoph (Hrsg): Handbuch Sprache in den Public Relations, S. 21-36. Wiesbaden: Springer Fachmedien Wiesbaden. https://doi.org/10.1007/978-3658-15745-6 2 .

Saxer, Ulrich (1999): Der Forschungsgegenstand der Medienwissenschaft. In: Leonhard, Joachim-Felix / Ludwig, Hans-Werner / Schwarze, Dietrich / Straßner, Erich (Hrsg.): Medienwissenschaft. Ein Handbuch zur Entwicklung der Medien und Kommunikationsformen. Band 1. Berlin, New York: Walter de Gruyter, 1-14.

Saxer, Ulrich (1980): Grenzen Der Publizistikwissenschaft. Wissenschaftswissenschaftliche Reflexionen Zur Zeitungs-/Publizistik-/Kommunikationswissenschaft Seit 1945. In: Publizistik 25, S. 525-543.

Schmidt, Jan (2009): Das neue Netz: Merkmale, Praktiken und Folgen des Web 2.0. Konstanz: UVK.

Schmidt, V. A. (2008): Discursive Institutionalism: The Explanatory Power of Ideas and Discourse. Annual Review of Political Science, 11, 303-326.

Scott, W. R. (2007). Institutions and Organizations: Ideas and Interests (3rd edition). Los Angeles: SAGE Publications, Inc.

Schulz, Wolfgang. 2018. Regulating Intermediaries to Protect Privacy Online - the Case of the German NetzDG. HIIG Discussion Paper Series, 2018-01. https://www.hiig.de/wp-content/uploads/2018/07/SSRN-id3216572.pdf

Taddicken, Monika/Schmidt, Jan-Hinrik (2017). Entwicklung und Verbreitung sozialer Medien. In Jan-Hinrik Schmidt/Monika Taddicken (Hrsg.), Handbuch Soziale Medien. Wiesbaden: Springer Fachmedien, S. 3-22. https://doi.org/10.1007/978-3-65803765-9 1

Van Dijck, José/Poell, Thomas (2013): Understanding Social Media Logic. In: Media \& Communication 1(1), S. 2-14. https://doi.org/10.12924/mac2013.01010002.

Zuckerberg, Marc (2018): A Blueprint for Content Governance and Enforcement. facebook.com, 15.11.2018. URL: https://perma.cc/YXC8-6XK4. 